\title{
CSF-1 and IL-34 levels in peri-implant crevicular fluid and saliva from patients having peri-implant diseases
}

\author{
Ronaldo Lira-Junior ${ }^{1,2}$ - Mayla K. S. Teixeira ${ }^{3}$ - Eduardo J. V. Lourenço ${ }^{3}$ - Daniel M. Telles $^{3}$. \\ Carlos Marcelo Figueredo ${ }^{2,4,5}$. Elisabeth A. Boström ${ }^{1}$ (D)
}

Received: 26 October 2018 / Accepted: 2 May 2019 / Published online: 17 May 2019

(C) The Author(s) 2019

\begin{abstract}
Objective Colony-stimulating factor (CSF)-1 and interleukin (IL)-34 are growth factors that regulate myeloid cell functions and support osteoclastogenesis. CSF-1 and IL-34 levels in peri-implant diseases are yet unknown. This study evaluated CSF-1, IL-34, and IL-1 $\beta$ levels in saliva and peri-implant crevicular fluid (PICF) from patients having mucositis or peri-implantitis, as well as their correlation to clinical parameters of disease.

Material and methods Forty-three patients were included (mean age 61.1 1 8.4; 62.8\% female), 20 having mucositis and 23 having peri-implantitis. Patients were clinically examined and unstimulated whole saliva and PICF were collected. Levels of CSF-1, IL-34, and IL-1 $\beta$ were determined by enzyme-linked immunosorbent assays.

Results CSF-1 levels were higher in PICF from peri-implantitis compared with mucositis patients $(p=0.028)$, whereas IL-34 levels showed no significant difference between the groups $(p=0.060)$. No significant difference was found in PICF IL-1 $\beta$ levels between the groups. Salivary levels of CSF-1 and IL-34 did not differ significantly between mucositis and peri-implantitis. No significant difference was observed in the salivary levels of IL-1 $\beta$ between groups $(p=0.061)$. CSF- 1 and IL-1 $\beta$ correlated significantly in both saliva and PICF. CSF-1 levels in saliva correlated with its levels in PICF. PICF CSF-1 levels showed potential to discriminate between peri-implantitis and mucositis (AUC $=0.695,95 \% \mathrm{CI} 0.53-0.85 ; p=0.029$ ).

Conclusion Increased levels of CSF-1 in peri-implant crevicular fluid, but not in saliva, were found in peri-implantitis patients, which might aid to discriminate the early and late stages of peri-implant diseases.

Clinical relevance This result suggests an increased osteoclastogenic potential in peri-implantitis patients.
\end{abstract}

Keywords Peri-implantitis $\cdot$ Interleukin-34 $\cdot$ Macrophage colony-stimulating factor $\cdot$ Saliva

Ronaldo Lira-Junior and Mayla K. S. Teixeira contributed equally to this work.

Elisabeth A. Boström

elisabeth.bostrom@ki.se

1 Department of Dental Medicine, Division of Oral Diseases, Karolinska Institute, Stockholm, Sweden

2 Department of Periodontology, Faculty of Odontology, Rio de Janeiro State University, Rio de Janeiro, Brazil

3 Department of Prosthodontics, Faculty of Odontology, Rio de Janeiro State University, Rio de Janeiro, Brazil

4 School of Dentistry and Oral Health, Griffith University, Gold Coast, QLD, Australia

5 Menzies Health Institute Queensland, Griffith University, Gold Coast, QLD, Australia

\section{Introduction}

Peri-implant diseases are highly prevalent conditions at a subject level, with $46.8 \%$ and $19.8 \%$ of the individuals with implants having mucositis and peri-implantitis, respectively [1]. Peri-implant mucositis is the inflammatory reaction in the soft tissues surrounding a functioning implant without loss of supporting bone. Peri-implantitis is characterized by loss of supporting bone associated with an inflammatory reaction around an implant in function, which may lead to implant loss $[2,3]$. Peri-implant diseases result from an imbalance between the microbiota and the host response [3]. The development of the disease is characterized by a dense inflammatory infiltrate in the connective tissue, in which polymorphonuclear leukocytes and macrophages are among the most abundant [4], suggesting these cells are involved in disease pathogenesis. 
Colony-stimulating factor (CSF)-1 is a pleiotropic growth factor that regulates survival, proliferation, and differentiation of myeloid cells. It acts in concert with receptor activator of nuclear factor kappa-B ligand (RANKL) to regulate the differentiation of mononuclear phagocytes to osteoclasts [5]. CSF-1 has been implicated in many inflammatory diseases [6, 7], including periodontal disease [8]. Interleukin (IL)-34 was identified as an alternative ligand for CSF1R and also regulates myeloid cell functions [9]. Both CSF-1 and IL-34 share functions; however, they are not equivalent and have complimentary roles [10]. Similarly to CSF-1, IL-34 is expressed in human gingival fibroblasts and its expression can be enhanced by IL-1 $\beta$ and tumor necrosis factor alpha (TNF- $\alpha$ ). IL-34 can substitute CSF-1 in RANKL-induced osteoclastogenesis [11]. Blockage of the CSF-1 receptor (CSF1R) has been shown to result in reduced alveolar bone loss [8]. We have previously reported CSF-1 and IL-34 are altered in saliva from periodontitis patients [12, 13]. However, their levels in peri-implant diseases have not been explored yet.

The analysis of biomarkers in peri-implant crevicular fluid (PICF) may aid in early diagnosis and determination of patient susceptibility, as well as contribute to the determination of the prognosis of affected implants and guide the selection of treatment approaches $[14,15]$. It has been shown that the levels of IL-1 $\beta$ in PICF may assist in the early diagnosis of periimplantitis, though no difference is found between the early and later stages of peri-implant disease [16]. Collection of crevicular fluid is time-consuming and technically demanding, and saliva is to a greater extent used to assess the potential of biomarkers to diagnose and monitor periodontal and periimplant diseases. However, it is needed to evaluate whether salivary levels of biomarkers reflect those detected in PICF, and to evaluate their relation to clinical parameters.

Therefore, since CSF-1 and IL-34 are involved in osteoclastogenesis, their investigation in peri-implant diseases might give us insights into the disease pathogenesis and reveal the potential diagnostic ability of these markers to differentiate between peri-implant mucositis and peri-implantitis. Consequently, this study aimed to assess the levels of CSF1 , IL-34, and IL-1 $\beta$ in saliva and PICF from patients having mucositis or peri-implantitis, as well as the correlations among the markers and to clinical parameters. In addition, this study evaluated the correlation between the salivary and PICF levels of CSF-1, IL-34, and IL-1 $\beta$.

\section{Material and methods}

\section{Participants}

This cross-sectional study enrolled 43 systemically healthy participants with osseointegrated implants (mean age 61.1 \pm 8.4 ; $62.8 \%$ female), as previously described in detail [17]. Briefly, all participants were partially edentulous with an implantretained prosthesis in function for at least 6 months. The mean $( \pm \mathrm{sd})$ functional time in use of the implants was $61.7 \pm$ 30.0 months. Patients were excluded if they were smokers, had received periodontal or peri-implant therapy in the preceding 6 months, were pregnant or breastfeeding, or had used medication, such as antibiotics and anti-inflammatory drugs, in the last 6 months.

One calibrated dentist performed all the clinical examination and determined the following parameters: visible plaque index (VPI), bleeding on probing (BOP), probing depth (PD), and clinical attachment level (CAL). Gingival index (GI) [20] was measured in the sites selected for PICF collection, as the GI allows the assessment of different grades of inflammation. The examinations were performed with a periodontal probe $(\mathrm{Hu}-$ Friedy, Chicago, IL, USA) in six sites per tooth, excluding the third molars. The intra-examiner calibration included 5 patients measured twice with an interval of $6 \mathrm{~h}$ between the examinations and the concordance within $\pm 1 \mathrm{~mm}$ was $97 \%$ for PD measurements. A full-mouth series of intraoral periapical radiographs was taken to aid the diagnosis and selection of sites for PICF collection. Twenty participants were diagnosed as having periimplant mucositis and 23 participants were diagnosed with periimplantitis. Mucositis was defined as the presence of clinically inflamed sites without any significant radiographic bone loss (bone loss around the implant not reaching the first thread), while peri-implantitis was defined as the presence of inflamed sites and bone loss involving at least two implant threads [18, 19]. The disease extent was calculated as the number of implants affected by disease.

\section{Peri-implant crevicular fluid and saliva collection}

PICF was collected from 2 to 3 inflamed sites that had gingival index of 1 or 2 [20]. Sites were isolated with cotton rolls, and the surfaces were gently dried with air. PICF was collected with paper strips (Periopaper, Oraflow, NY, USA), which were introduced into the peri-implant crevice for up to $30 \mathrm{~s}$. Samples contaminated with blood were discarded. Volume of PICF was measured in the Periotron (Periotron ${ }^{\circledR} 8000$, Oraflow, NY, USA), and the paper strips were pooled and eluted in $200 \mu \mathrm{l}$ of phosphate-buffered saline for $45 \mathrm{~min}$. Protease inhibitor was added to all samples (Sigma-Aldrich, St. Louis, MO, USA). Paper strips were removed, and the sample was centrifuged at $3000 \mathrm{~g}$ for $5 \mathrm{~min}$ and the supernatant stored at $-70{ }^{\circ} \mathrm{C}$ until analysis.

Unstimulated whole saliva was also collected. Patients refrained from eating and drinking and did not perform any oral hygiene measures for at least $1 \mathrm{~h}$ prior to collection. Before collection, each participant rinsed their mouth with tap water, and after $5 \mathrm{~min}$, participants expectorated into sterile tubes until $1 \mathrm{ml}$ was reached. Protease inhibitor was added to all saliva 
samples. Samples were centrifuged at $3000 \mathrm{~g}$ for $5 \mathrm{~min}$, and then the supernatant was stored at $-70{ }^{\circ} \mathrm{C}$ until analysis.

\section{CSF-1, IL-34, and IL-1 $\beta$ immunoassays}

Levels of CSF-1, IL-34, and IL- $1 \beta$ were determined using commercial enzyme-linked immunosorbent assays according to the manufacturer's instructions (R\&D Systems, Minneapolis, MN, USA). Readings were made using a microplate spectrophotometer (SpectraMAX 340, Sunnyvale, CA, USA) with wavelength set at $450 \mathrm{~nm}$ and correction set to $540 \mathrm{~nm}$ to subtract background. Samples below the limit of detection were set as 0 .

\section{Statistical analysis}

Data analyses were performed using Statistical Package for Social Sciences (SPSS), version 24 (IBM Corporation, Armonk, NY, USA). Continuous variables are presented as mean and standard deviation (SD) or median (interquartile range), and categorical variables as frequencies. Data normality was evaluated by the Shapiro-Wilk test. Mann-Whitney test was used to compare the continuous variables between the two groups. Chi-square test was used to compare frequencies between the groups. Spearman correlation coefficient was calculated to assess the relation between clinical variables and biomarker levels. Biomarker fold-changes were calculated by dividing the means of the two study groups. Statistical significance was set at $p \leq 0.05$.

To evaluate the biomarkers' ability to discriminate periimplantitis patients from mucositis patients, area under the receiver-operating characteristic curve (AUROC) and 95\% confidence intervals $(\mathrm{CI})$ were calculated for the markers showing a significant difference between the groups.

\section{Results}

\section{Clinical parameters}

There was no significant difference between the groups with respect to age or gender. Peri-implantitis patients presented higher full mouth mean of PD in teeth $(p=0.036)$, as well as higher PD mean in implants $(p=0.003)$ compared with mucositis patients. The number of implants did not differ significantly between groups $(p=0.075)$. Periodontal and periimplant clinical parameters are presented in Table 1. Out of the 43 patients included in the study, 23 presented concomitant periodontitis $(\mathrm{PD} \geq 5 \mathrm{~mm}$ and $\mathrm{CAL} \geq 3 \mathrm{~mm}$ ).

\section{CSF-1, IL-34, and IL-1 $\beta$ levels in saliva and PICF}

CSF-1 and IL-34 were detected in $76.7 \%$ and $60.5 \%$ of the PICF samples, respectively. In saliva, CSF-1 and IL-34 were
Table 1 Demographics and peri-implant and periodontal parameters of the two study groups

\begin{tabular}{lccc}
\hline Variable & $\begin{array}{l}\text { Mucositis } \\
(n=20)\end{array}$ & Peri-implantitis $(n=23)$ & $p$ value* \\
\hline Age (years) & $61.5( \pm 10.8)$ & $60.8( \pm 5.9)$ & 0.574 \\
Gender (male/female) & $8 / 12$ & $8 / 15$ & 0.724 \\
Full mouth periodontal parameters & & \\
Number of teeth & $19.0(4.7)$ & $17.0(7.0)$ & 0.085 \\
VPI (\%) & $76.0(32.0)$ & $69.0(36.0)$ & 0.165 \\
BOP (\%) & $34.0(38.7)$ & $35.0(20.0)$ & 0.697 \\
PD (mm) & $2.1(0.5)$ & $2.3(0.3)$ & 0.036 \\
CAL (mm) & $1.5(0.7)$ & $1.6(1.4)$ & 0.961 \\
Full-mouth peri-implant parameters & & \\
Number of implants & $4.0(1.0)$ & $6.0(5.0)$ & 0.075 \\
VPI (\%) & $25.0(39.9)$ & $40.0(25.0)$ & 0.676 \\
BOP (\%) & $60.0(22.9)$ & $55.5(46.5)$ & 0.695 \\
PD (mm) & $2.6(0.5)$ & $3.1(0.7)$ & 0.003 \\
\hline
\end{tabular}

Data are presented as median (interquartile range) or frequencies. Age is presented as mean ( \pm standard deviation). $* p$ value was evaluated using Mann-Whitney or chi-square test. VPI visible plaque index, $B O P$ bleeding on probing, $P D$ probing depth, $C A L$ clinical attachment loss. Italic $\mathrm{p}$ values indicates statistical significance

detected in $100 \%$ and $97.7 \%$, respectively. IL- $1 \beta$ was detected in all saliva and PICF samples.

Mean levels of CSF-1 were 1.35- and 3.78-fold higher in saliva and PICF from peri-implantitis patients compared with mucositis patients, respectively. PICF levels of CSF-1 showed a significant difference between the groups $(p=0.028$; Fig. 1b), whereas salivary levels did not differ significantly between the groups $(p=0.770$; Fig. 1a). Peri-implantitis patients showed 1.33- and 3.83-fold higher mean levels of IL-34 in saliva and PICF than mucositis patients, respectively; however, these differences did not reach statistical significance $(p=0.401, p=0.060$; Fig. 1$)$.

Salivary levels of IL-1 $\beta$ did not differ significantly between patients with peri-implantitis and those with mucositis ( $p=0.061$; Fig. 1a). No significant difference was found between the groups for IL- $1 \beta$ levels in PICF $(p=0.944)$. Regarding the concomitant presence of periodontitis, no significant difference was seen between those with and without periodontitis for the salivary and PICF levels of CSF-1, IL-34, and IL-1 $\beta$ (data not shown).

\section{Correlation analyses}

Correlations between clinical parameters, and the levels of CSF-1, IL-34, and IL-1 $\beta$ in saliva and PICF, are presented in Fig. 2a, and correlations among the markers are presented in Fig. 2b. No significant correlation was found between the salivary levels of the markers and the clinical parameters. IL34 levels in PICF correlated significantly with BOP and with 
a Saliva
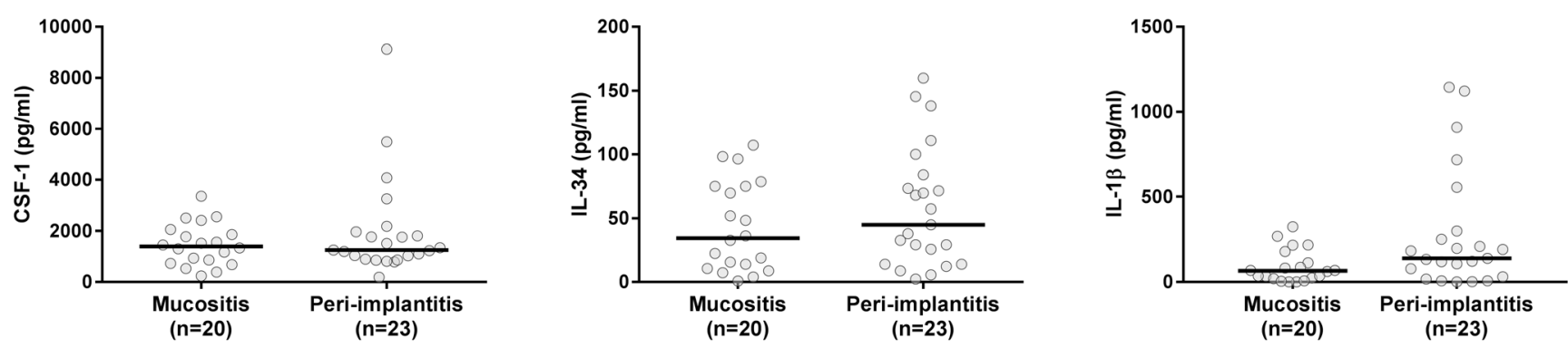

b PICF
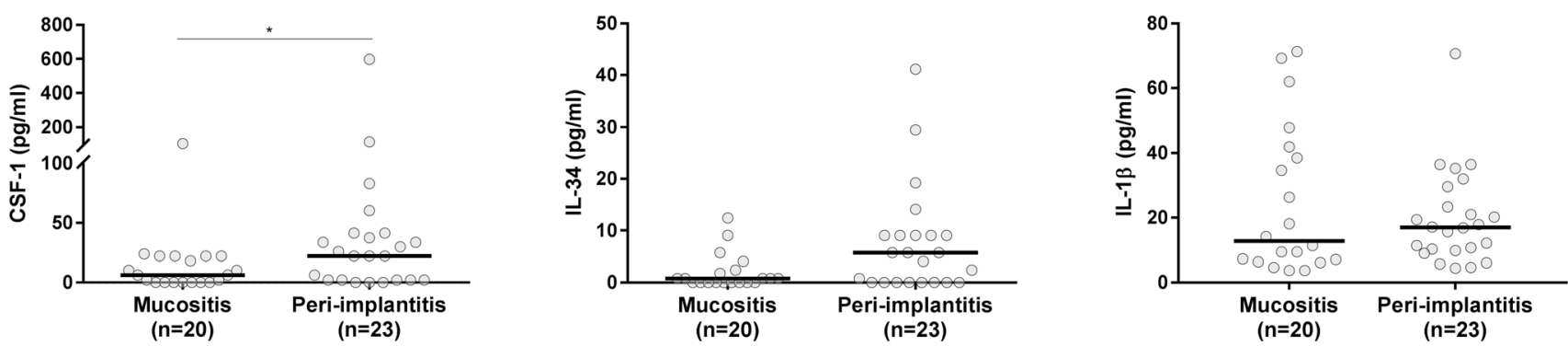

Fig. 1 Levels of CSF-1, IL-34, and IL-1 $\beta$ in saliva (a) and in peri-implant crevicular fluid (b) from individuals with peri-implant mucositis and periimplantitis. $* p<0.05$ (Mann-Whitney test)

the disease extent in implants, whereas IL- $1 \beta$ levels correlated with VPI in teeth. Levels of IL-1 $\beta$ in PICF also correlated significantly with age.

Salivary levels of CSF-1 correlated significantly with its levels in PICF. Neither IL-34 nor IL-1 $\beta$ levels in saliva correlated significantly with their corresponding levels in PICF. In both saliva and PICF, CSF-1 levels correlated significantly with the levels of IL- $1 \beta$.

\section{Diagnostic ability of CSF-1}

Since CSF-1 levels in PICF showed a significant difference between the groups, its ability to differentiate the groups was assessed. CSF-1 levels showed an AUROC of 0.695 (95\% CI $0.53-0.85 ; p=0.029)$ to discriminate patients having periimplantitis from those having mucositis.

\section{Discussion}

This study is, to the best of our knowledge, the first to assess the levels of the macrophage growth factors CSF-1 and IL-34 in matched PICF and saliva from individuals with peri-implant diseases. It is important to understand the immunological differences between inflamed sites without tissue destruction and those with tissue destruction, to comprehend what factors are more relevant for bone loss rather than to inflammation itself. We found increased levels of CSF-1 in PICF from peri-implantitis patients with diagnostic potential of CSF-1 to discriminate between peri-implantitis and mucositis patients. These findings imply involvement of CSF-1 in the pathogenesis of periimplantitis, which deserves further investigation.

We report a significant increase in the CSF-1 levels in PICF, but not in saliva, from peri-implantitis patients as compared to peri-implant mucositis. Measuring CSF-1 in PICF might offer a diagnostic ability to differentiate periimplantitis from mucositis as evidenced by an AUROC of 0.695 (95\% CI 0.53-0.85). These two conditions presented peri-implant mucosa inflammation and were differentiated clinically by the presence of bone loss beyond bone remodeling. This difference is important to determine since early intervention and removal of etiology will reverse mucositis [19, 21]. A positive correlation between CSF- 1 and IL- $1 \beta$ levels in both saliva and PICF was seen, which is in accordance with previous work from our group [12]. CSF-1 regulates the production, maintenance, and function of cells from the mononuclear phagocyte lineage, as well as the function of other cells expressing the CSF-1R [5]. CSF-1 is expressed by gingival fibroblasts and takes part in RANKL-stimulated osteoclastogenesis [11]. CSF-1 levels in saliva have been shown to be increased in individuals with periodontitis $[12,13]$. However, the levels of CSF-1 in saliva were higher in periodontitis when compared to healthy, but not when compared to gingivitis [13]. This result is in line with the finding of similar levels between peri-implantitis and mucositis. Interestingly, CSF-1 levels in PICF correlated significantly with its levels in saliva, which suggests crevicular fluid as an important source of this growth factor in saliva. 
a

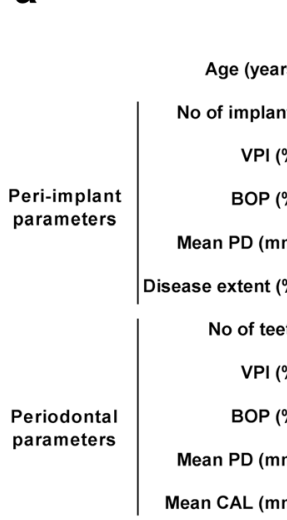

Saliva

\begin{tabular}{|c|c|c|}
\hline CSF-1 & IL-34 & IL- $1 \beta$ \\
\hline
\end{tabular}
(1)

\section{.} sti
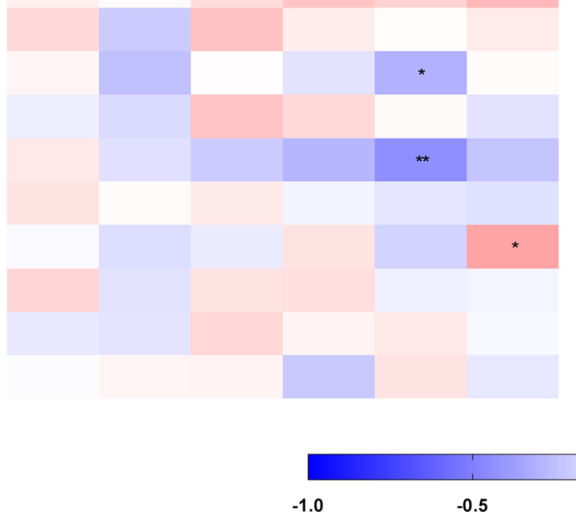

b

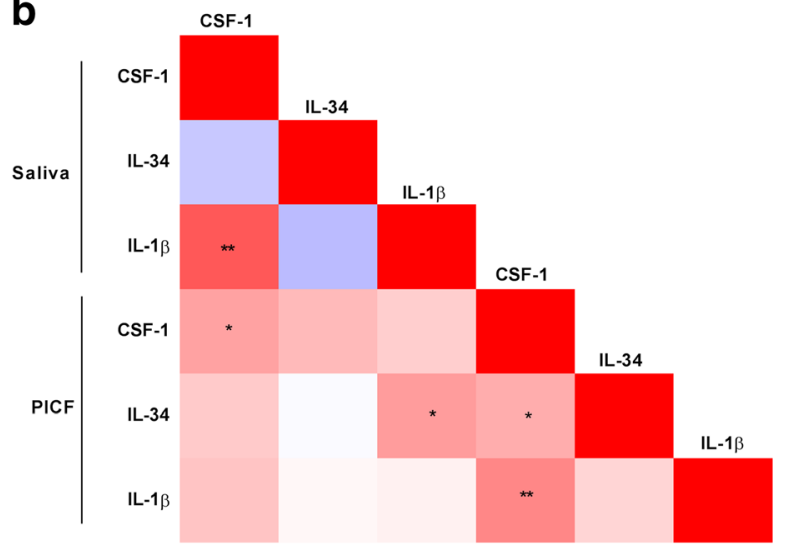

Fig. 2 Heat maps exhibiting correlations between the biomarkers and clinical parameters (a) and among the biomarkers (b) in saliva and peri-implant crevicular fluid. $* p<0.05, * * p<0.01$ (Spearman correlation)

The levels of IL-34 in saliva did not exhibit significant differences between peri-implantitis and mucositis; however, it showed 3.8-fold higher PICF levels in peri-implantitis without reaching statistical significance. Our results are partially in line with previous reports on elevated levels of IL-34 in GCF from individuals having chronic periodontitis [22, 23]. PICF levels of IL-34 did not correlate significantly with its levels in saliva; however, IL-34 levels in GCF have been reported to positively correlate to its level in plasma which suggests contribution of IL-34 from other sources than from crevicular fluid into saliva [22]. Furthermore, we have previously measured elevated salivary levels of IL-34 in periodontally healthy individuals compared with both gingivitis and periodontitis patients; however, no significant difference was seen between gingivitis and periodontitis patients [13]. In the current study, we did not include participants with healthy implants, making a direct comparison with our previous findings in saliva difficult.

The cellular sources of IL-34 and secretion into different biological fluids in homeostasis and inflammation deserves investigation. IL-34 has been shown to be potentially pathogenic in other inflammatory diseases, such as rheumatoid arthritis and inflammatory bowel disease [10, 24, 25]. Measurement of IL-34 in serum has been shown to predict radiographic progression in patients with rheumatoid arthritis [26]. IL-34 is expressed by gingival fibroblasts, and its expression can be enhanced by pro-inflammatory stimuli, such as IL$1 \beta$ and TNF- $\alpha$. Also, IL-34 can substitute CSF-1 in RANKLinduced osteoclastogenesis, suggesting a role in inflammation-driven bone resorption [11]. Blockage of the CSF-1 receptor resulted in decreased bone resorption in an experimental model of periodontitis [8]. However, both IL34 and CSF-1 can signal through the CSF-1 receptor. It is well-known that CSF1R-deficient mice present a more severe phenotype than that of mice lacking CSF-1, underlining that IL-34 and CSF-1 have distinct biological functions [27]. The specific roles of IL-34 and CSF-1 in peri-implant/periodontal bone loss deserves further investigation.

We found no significant difference in PICF levels of IL- $1 \beta$ between peri-implantitis and mucositis. Also, its salivary levels did not show a significant difference between the groups. IL- $1 \beta$ is one of the most studied cytokines in periimplant diseases; however, results regarding its relation to peri-implant diseases have been contradictory. A systematic review has shown increased levels in PICF in both periimplantitis and mucositis patients in comparison with healthy participants; however, in agreement with our result, IL-1 $\beta$ levels were not significantly altered when peri-implantitis was compared to mucositis [16]. Regarding saliva, higher levels of IL- $1 \beta$ were found in male participants with periimplantitis compared with participants without [28], whereas no significant difference in salivary levels of IL-1 $\beta$ was reported between totally edentulous participants with periimplantitis and those with mucositis [29]. The relationship between salivary levels of IL-1 $\beta$ and peri-implant inflammation deserves further consideration.

Our results should be interpreted having in mind the limitations of the study. Its cross-sectional nature does not allow any causal claim to be made. Prospective studies with larger cohorts would provide great insights into the utility of CSF-1 and IL-34 macrophage growth factors to diagnose and monitor peri-implant diseases. Also, this study did not explore the involvement of the macrophage growth factors in peri-implant disease pathogenesis or address the cellular origin of IL-34 and CSF-1 in PICF and saliva, which deserves further investigation. Despite that, a strength of the study is the age matching between groups, given that age has been shown to impact on the immune response, and that salivary levels of 
CSF-1 have been correlated with age [12]. Furthermore, the evaluation of matched PICF and saliva enabled us to assess whether saliva reflected the inflammatory status of pooled selected sites, which is a strength of our study.

In conclusion, this study reports higher levels of CSF-1 in peri-implant crevicular fluid, but not in saliva, in individuals having peri-implantitis, which might provide diagnostic value to discriminate peri-implantitis from mucositis. An altered osteoclastogenic potential is suggested in peri-implantitis.

\section{Compliance with ethical standards}

Conflict of interest The authors declare that they have no conflict of interest.

Ethical approval The study was approved by the Ethics Committee of the Pedro Ernesto University Hospital (No 857.118). All procedures performed in the study were in accordance with the 1964 Helsinki declaration and its later amendments.

Informed consent All participants gave their informed consent prior to inclusion in the study.

Open Access This article is distributed under the terms of the Creative Commons Attribution 4.0 International License (http:// creativecommons.org/licenses/by/4.0/), which permits unrestricted use, distribution, and reproduction in any medium, provided you give appropriate credit to the original author(s) and the source, provide a link to the Creative Commons license, and indicate if changes were made.

\section{References}

1. Lee CT, Huang YW, Zhu L, Weltman R (2017) Prevalences of periimplantitis and peri-implant mucositis: systematic review and metaanalysis. J Dent 62:1-12. https://doi.org/10.1016/j.jdent.2017.04.011

2. Zitzmann NU, Berglundh T (2008) Definition and prevalence of peri-implant diseases. J Clin Periodontol 35:286-291. https://doi. org/10.1111/j.1600-051X.2008.01274.X

3. Heitz-Mayfield LJ, Lang NP (2010) Comparative biology of chronic and aggressive periodontitis vs. peri-implantitis. Periodontol 2000 53: 167-181. https://doi.org/10.1111/j.1600-0757.2010.00348.x

4. Nguyen Vo TN, Hao J, Chou J, Oshima M, Aoki K, Kuroda S, Kaboosaya B, Kasugai S (2017) Ligature induced peri-implantitis: tissue destruction and inflammatory progression in a murine model. Clin Oral Implants Res 28:129-136. https://doi.org/10.1111/clr.12770

5. Pixley FJ, Stanley ER (2004) CSF-1 regulation of the wandering macrophage: complexity in action. Trends Cell Biol 14:628-638. https://doi.org/10.1016/j.tcb.2004.09.016

6. Campbell IK, Rich MJ, Bischof RJ, Hamilton JA (2000) The colony-stimulating factors and collagen-induced arthritis: exacerbation of disease by M-CSF and G-CSF and requirement for endogenous M-CSF. J Leukoc Biol 68:144-150

7. Marshall D, Cameron J, Lightwood D, Lawson AD (2007) Blockade of colony stimulating factor-1 (CSF-I) leads to inhibition of DSS-induced colitis. Inflamm Bowel Dis 13:219-224. https:// doi.org/10.1002/ibd.20055

8. Kimura K, Kitaura H, Fujii T, Ishida M, Hakami ZW, TakanoYamamoto T (2014) An anti-c-Fms antibody inhibits osteoclastogenesis in a mouse periodontitis model. Oral Dis 20:319-324. https://doi.org/10.1111/odi.12117

9. Lin H, Lee E, Hestir K, Leo C, Huang M, Bosch E, Halenbeck R, Wu G, Zhou A, Behrens D, Hollenbaugh D, Linnemann T, Qin M, Wong J, Chu K, Doberstein SK, Williams LT (2008) Discovery of a cytokine and its receptor by functional screening of the extracellular proteome. Science 320:807-811. https://doi.org/10.1126/science. 1154370

10. Guillonneau C, Bezie S, Anegon I (2017) Immunoregulatory properties of the cytokine IL-34. Cell Mol Life Sci 74:2569-2586. https://doi.org/10.1007/s00018-017-2482-4

11. Bostrom EA, Lundberg P (2013) The newly discovered cytokine IL-34 is expressed in gingival fibroblasts, shows enhanced expression by proinflammatory cytokines, and stimulates osteoclast differentiation. PLoS One 8:e81665. https://doi.org/10.1371/journal.pone.0081665

12. Lira-Junior R, Akerman S, Gustafsson A, Klinge B, Bostrom EA (2017) Colony stimulating factor-1 in saliva in relation to age, smoking, and oral and systemic diseases. Sci Rep 7:7280. https:// doi.org/10.1038/s41598-017-07698-4

13. Martinez GL, Majster M, Bjurshammar N, Johannsen A, Figueredo CM, Bostrom EA (2017) Salivary colony stimulating factor- 1 and interleukin-34 in periodontal disease. J Periodontol 88:e140-e149. https://doi.org/10.1902/jop.2017.170081

14. Petkovic AB, Matic SM, Stamatovic NV, Vojvodic DV, Todorovic TM, Lazic ZR, Kozomara RJ (2010) Proinflammatory cytokines (IL-1beta and TNF-alpha) and chemokines (IL-8 and MIP-1alpha) as markers of peri-implant tissue condition. Int J Oral Maxillofac Surg 39:478-485. https://doi.org/10.1016/j.ijom.2010.01.014

15. Zani SR, Moss K, Shibli JA, Teixeira ER, de Oliveira Mairink R, Onuma T, Feres M, Teles RP (2016) Peri-implant crevicular fluid biomarkers as discriminants of peri-implant health and disease. $\mathrm{J}$ Clin Periodontol 43:825-832. https://doi.org/10.1111/jcpe.12586

16. Faot F, Nascimento GG, Bielemann AM, Campao TD, Leite FR, Quirynen M (2015) Can peri-implant crevicular fluid assist in the diagnosis of peri-implantitis? A systematic review and meta-analysis. $\mathrm{J}$ Periodontol 86:631-645. https://doi.org/10.1902/jop.2015.140603

17. Teixeira MKS, Lira-Junior R, Telles DM, Lourenco EJV, Figueredo CM (2017) Th17-related cytokines in mucositis: is there any difference between peri-implantitis and periodontitis patients? Clin Oral Implants Res 28:816-822. https://doi.org/10.1111/clr.12886

18. Lindhe J, Meyle J, Group D of European Workshop on Periodontology (2008) Peri-implant diseases: consensus report of the sixth European workshop on periodontology. J Clin Periodontol 35:282-285. https:// doi.org/10.1111/j.1600-051X.2008.01283.x

19. American Academy of Periodontology (2013) Peri-implant mucositis and peri-implantitis: a current understanding of their diagnoses and clinical implications. J Periodontol 84:436-443. https://doi.org/ 10.1902/jop.2013.134001

20. Loe H, Silness J (1963) Periodontal disease in pregnancy. I. Prevalence and severity. Acta Odontol Scand 21:533-551

21. Pontoriero R, Tonetti MP, Carnevale G, Mombelli A, Nyman SR, Lang NP (1994) Experimentally induced peri-implant mucositis. A clinical study in humans. Clin Oral Implants Res 5:254-259

22. Guruprasad CN, Pradeep AR (2018) Interleukin-34 levels in gingival crevicular fluid and plasma in periodontal health and disease with and without type-2 diabetes mellitus. J Investig Clin Dent 9: e12317. https://doi.org/10.1111/jicd.12317 
23. Guruprasad CN, Pradeep AR (2018) Effect of nonsurgical periodontal therapy on interleukin-34 levels in periodontal health and disease. Indian J Dent Res 29:280-285. https://doi.org/10.4103/ijdr.IJDR_ 527_17

24. Zhang L, Cui M, Ding L, Xia L, Lu J, Shen H (2018) Interleukin-34 aggravates the severity of arthritis in collagen-induced arthritis mice by inducing interleukin-17 production. J Interf Cytokine Res 38: 221-225. https://doi.org/10.1089/jir.2017.0095

25. Zwicker S, Martinez GL, Bosma M, Gerling M, Clark R, Majster M, Soderman J, Almer S, Bostrom EA (2015) Interleukin 34: a new modulator of human and experimental inflammatory bowel disease. Clin Sci (Lond) 129:281-290. https://doi.org/10.1042/ CS20150176

26. Chang SH, Choi BY, Choi J, Yoo JJ, Ha YJ, Cho HJ, Kang EH, Song YW, Lee YJ (2015) Baseline serum interleukin-34 levels independently predict radiographic progression in patients with rheumatoid arthritis. Rheumatol Int 35:71-79. https://doi.org/10.1007/ s00296-014-3056-5
27. Nakamichi Y, Udagawa N, Takahashi N (2013) IL-34 and CSF-1: similarities and differences. J Bone Miner Metab 31:486-495. https://doi.org/10.1007/s00774-013-0476-3

28. Al-Askar M, Ajlan S, Alomar N, Al-Daghri NM (2018) Clinical and radiographic peri-implant parameters and whole salivary interleukin-1beta and interleukin-6 levels among type- 2 diabetic and nondiabetic patients with and without peri-implantitis. Med Princ Pract 27:133-138. https://doi.org/10.1159/000488032

29. Fonseca FJ, Moraes Junior M, Lourenco EJ, Teles Dde M, Figueredo CM (2014) Cytokines expression in saliva and periimplant crevicular fluid of patients with peri-implant disease. Clin Oral Implants Res 25:e68-e72. https://doi.org/10.1111/clr.12052

Publisher's note Springer Nature remains neutral with regard to jurisdictional claims in published maps and institutional affiliations. 